\title{
Diagnosis and Management of Cryptococcal Relapse
}

\author{
Abdu K Musubire ${ }^{1}$, David R Boulware ${ }^{2}$, David B Meya ${ }^{1,2}$ and Joshua Rhein ${ }^{2 *}$
}

${ }^{1}$ Infectious Disease Institute, Makerere University, Kampala, Uganda

${ }^{2}$ University of Minnesota, Minneapolis, MN, USA

\begin{abstract}
Despite improvements in the antifungal regimens and the roll out of antiretroviral therapy (ART) in subSaharan Africa, mortality due to cryptococcal meningitis remains high. Relapse of an initially successfully treated infection contributes to this mortality and is often a clinical dilemma in differentiating between paradoxical immune reconstitution inflammatory syndrome (IRIS) and culture-positive relapse or treatment failure. Herein, we present a clinical case scenario and review the case definitions, differential diagnosis, and management of relapse with an emphasis on the current diagnostic and management strategies. We also highlight the challenges of resistance testing and management of refractory relapse cases. The risk of relapse is influenced by: 1) the choice of induction therapy, with higher mortality risk with fluconazole monotherapy which can select for resistance; 2 ) non-adherence to or lack of secondary prophylaxis; 3 ) failure of linkage-to-care or retention-in-care of HIV ART programs.
\end{abstract}

Keywords: HIV; Cryptococcal meningitis; Cryptococcus; Relapse; Drug resistance

\section{Introduction}

Cryptococcal meningitis(CM) is a relatively common opportunistic infection in human immunodeficiency virus (HIV)infected persons and can also occur in persons with other causes of impaired immunity as well as persons without any apparent underlying immune deficiency $[1,2]$. Cryptococcal meningitis has emerged as the second leading cause of infectious morbidity and mortality in persons living with acquired immunodeficiency syndrome (AIDS [3,4]. Despite improvements in the antifungal regimens and the roll out of antiretroviral therapy (ART) [5-8], cryptococcal-related mortality remains high, especially in subSaharan Africa [9-12]. Relapse of an initially successfully treated infection contributes to this mortality and is often a clinical dilemma [13-15]. A case presentation and a discussion of common clinical issues are presented.

\section{Case presentation}

A 24 year old Ugandan man with HIV presented to an urban outpatient clinic with worsening headache over one week. The patient had a recent history of cryptococcal meningitis (CM) which had been diagnosed 4 months prior. While initially hospitalized for CM, the patient had received 14 consecutive days of amphotericin along with $800 \mathrm{mg} /$ day fluconazole and serial lumbar punctures (LP) to alleviate the elevated intracranial pressure. Cerebrospinal fluid (CSF) white blood cell count (WBC) was $<3$ cells $/ \mu \mathrm{L}$. The CSF opening pressure and quantitative cryptococcal colonies from serial lumber punctures are summarized in Table 1. From his initial culture, the fluconazole minimum inhibitory concentration (MIC) was measured as $32 \mu \mathrm{g} /$ $\mathrm{mL}$ (dose-dependent sensitivity), and the fluconazole dose was increased to $1200 \mathrm{mg} /$ day. The patient improved steadily throughout their two week hospitalization, although his hospitalization was complicated by a persistent cough, amphotericin infusion-related thrombophlebitis, and fevers due to gram-negative bacteremia that resolved with intravenous antibiotics. An evaluation for pulmonary tuberculosis (TB) was undertaken with Xpert MTB/RIF (Cepheid, Inc.) and was negative.

He had been first diagnosed with HIV two years previously, and his nadir CD4 count was 4 cells/ $\mu \mathrm{L}$ with a viral load of 230,000

\begin{tabular}{|c|c|c|}
\hline Day & Opening Pressure $\left(\mathrm{mm} \mathrm{H}_{2} \mathrm{O}\right)$ & Quantitative Colony Count $(\mathrm{CFU} / \mathrm{mL})$ \\
\hline 1 & 460 & $4.5 \times 10^{6}$ \\
\hline 3 & 440 & $8.4 \times 10^{3}$ \\
\hline 7 & 320 & $6.6 \times 10^{2}$ \\
\hline 14 & 180 & No growth \\
\hline
\end{tabular}

Table 1: Initial CSF Parameters of Patient in Case Presentation

copies/mL. He had started an antiretroviral therapy (ART) regimen of lamivudine, zidovudine, and efavirenz for 3 prior months and was on trimethoprim-sulfamethoxazole (TMP-SMX) prophylaxis. The fluconazole had been decreased from $800 \mathrm{mg} / \mathrm{day}$ to $400 \mathrm{mg} / \mathrm{day}$ after 1 month when the 2-week culture was known to be negative. The fluconazole was switched to secondary prophylaxis after 3 months to $200 \mathrm{mg} /$ day. He was doing well since hospital discharge until developing his current complaints of worsening headache.

What is in the differential diagnosis given the above information? What is the difference between disease relapse and treatment failure? Could this be an immune reconstitution inflammatory syndrome (IRIS)? What tests could be obtained to help differentiate between relapse, treatment failure, and IRIS?

The nomenclature of "relapse" varies widely. Clinically, there is an important distinction between symptomatic relapse and microbiologic relapse as one can be encountered without the other. For example, persons presenting with classic paradoxical cryptococcal-related Immune Reconstitution Inflammatory Syndrome (CM-IRIS) will have symptomatic relapse without microbiologic relapse [16]. While several definitions have previously been applied, relapse of cryptococcal meningitis generally and in the strictest sense implies 1) recurrence of symptoms, with 2) recovery of viable organisms

*Corresponding authors: Joshua Rhein, University of Minnesota, A-620 Mayo, MMC 250, 420 Delaware Street SE, Minneapolis, MN, USA, Tel: 612-626-9943; E-mail: rhei0005@umn.edu

Received March 04, 2013; Accepted April 24, 2013; Published April 29, 2013

Citation: Musubire AK, Boulware DR, Meya DB, Rhein J (2013) Diagnosis and Management of Cryptococcal Relapse. J AIDS Clinic Res S3: 003. doi:10.4172/21556113.S3-003

Copyright: (c) 2013 Musubire AK, et al. This is an open-access article distributed under the terms of the Creative Commons Attribution License, which permits unrestricted use, distribution, and reproduction in any medium, provided the original author and source are credited. 
from previously sterile cerebrospinal fluid (CSF) [16-18]. It is similarly important to determine past CSF culture status, as the mechanisms that lead to microbiologic relapse or persistent infection are distinct. Figure 1 summarizes the different processes that can lead to symptomatic relapse of cryptococcal meningitis.

\section{Relapse vs Persistent Cryptococcal Infection}

Microbiologic relapse is distinct from persistent infection (i.e. treatment failure), in which sterility of cerebrospinal fluid has never been achieved. In reality, this is a distinction that often cannot be done, from lack of data because persons with very low culture growth in their CSF are often remarkably asymptomatic, and these persons are often reluctant to have follow-up confirmatory LPs performed. Nevertheless, it is critical to confirm CSF culture sterility before reverting to fungistatic dosing of fluconazole at $400 \mathrm{mg} /$ day [19]. We routinely continue fluconazole at $800 \mathrm{mg} /$ day through the first $2-4$ weeks of consolidation therapy until the CSF is known to be sterile and ART is initiated.

The Infectious Disease Society of America (IDSA) differentiates relapse from persistent infection by defining persistent infection as positive CSF cultures after 4 weeks of proven antifungal therapy at an established effective doses [18]. The IDSA further emphasizes the importance of differentiating the relapse from persistent infection because changing cryptococcal antigen (CRAG) titers, presence of positive India ink examination results, and abnormal cellular reactions or chemistries are insufficient to diagnose microbiological relapse with an implied need to alter antifungal treatment strategies. Culture is essential for distinguishing, and quantitative cultures are even more informative. Most cases of relapse are attributable to inadequate primary therapy (dose and/or duration) or failure of compliance with consolidation or maintenance of fluconazole dose $[14,20]$.

Persistent infection should immediately raise concern for the presence of fluconazole resistance [14] where susceptibility testing is important. Fluconazole resistance should be considered when the minimum inhibitory concentration (MIC) is $\geq 16 \mu \mathrm{g} / \mathrm{mL}$ or if a threefold increase in MIC is observed over the treatment course, in which settings alternative treatment strategies should be considered. MIC cutoff values for $C$. neoformans are based on minimal clinical data, though isolates with an MIC of $\leq 8 \mu \mathrm{g} / \mathrm{mL}$ are generally considered susceptible (S), while dose-dependent resistance (S-DD) should be considered with an MIC of $16-32 \mu \mathrm{g} / \mathrm{mL}$, and resistance (R) should be assumed with an MIC of $\geq 64 \mu \mathrm{g} / \mathrm{mL}$ [21].

The major risk factor for the development of resistance is the use of low-dose fluconazole as induction monotherapy. The fungicidal effect of amphotericin minimizes this risk, and resistance remains low in areas where widespread amphotericin-based induction therapy has been adopted. A switch from fluconazole monotherapy to amphotericin as initial treatment for cryptococcal meningitis, for example, has essentially eliminated the majority (but not all) cases with secondary fluconazole resistance [20].

The effect that primary or secondary fluconazole prophylaxis has on the development of resistance in C. neoformans is less conclusive, though studies investigating the link between fluconazole prophylaxis and the risk of developing resistance in Candida may provide some insight. A systemic review of randomized trials in Candida concluded that fluconazole prophylaxis increased the risk for colonization with S-DD or resistant non-C. albicans species, but prophylaxis did not significantly affect the risk for invasive infections [22]. The risk for fungal breakthrough infections remains a concern that needs to be addressed in large prospective studies.

\section{Relapse vs. CM-IRIS}

When a patient develops recurrent symptoms of meningitis, it is important to consider both IRIS and relapse in the differential diagnosis [14,23]. It is clinically impossible to distinguish between IRIS and relapse based on symptoms alone. The critical distinguishing feature is a sterile CSF, which is more associated with paradoxical cryptococcal IRIS, rather than relapse [23,24]. Table 2 lists the criteria for paradoxical IRIS and potential alternative diagnoses. It is important to differentiate between IRIS and CM relapse because the therapeutic approach differs depending on the etiology behind recurrent symptoms. Unfortunately, it could take up to 1-2 weeks to obtain a final CSF culture result during low fungal burdens of

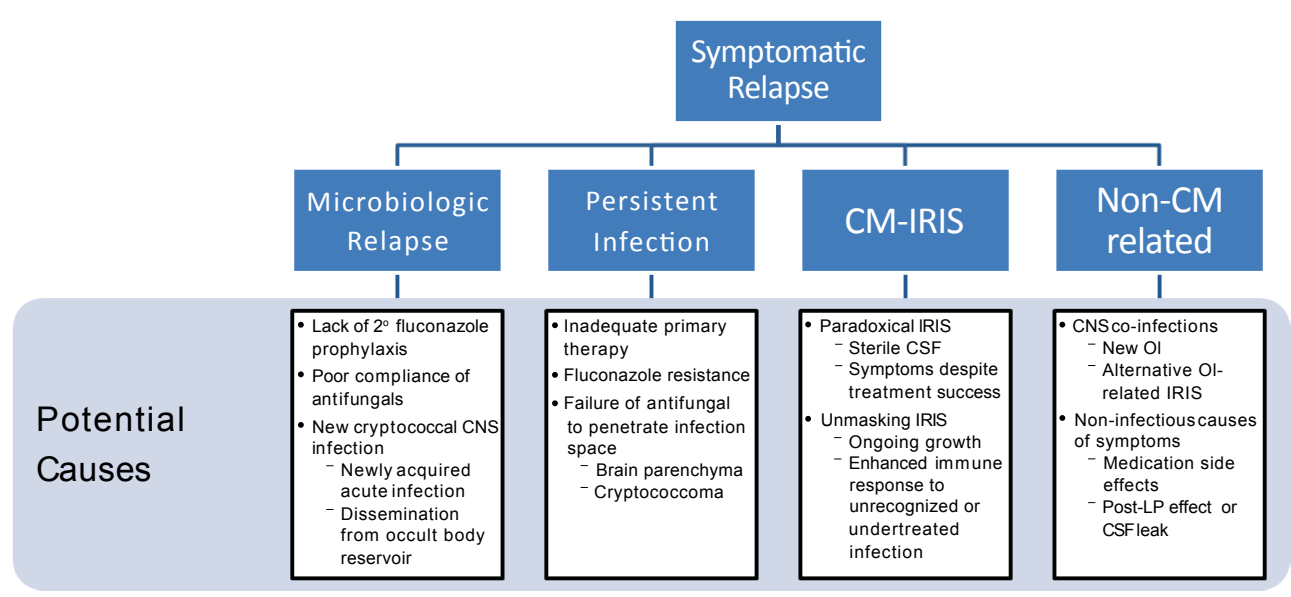

Figure 1: Differential Diagnosis for Symptomatic Relapse of Cryptococcal Meningitis

Differential diagnosis for persons presenting with recurrent symptoms of cryptococcal meningitis.

Pathologic mechanisms that can lead to symptomatic relapse of cryptococcal meningitis include microbiologic relapse, persistent infection, immune reconstitution inflammatory syndrome (IRIS), or unrelated causes that can mimic cryptococcal meningitis. Potential causes of each pathologic mechanism are listed and discussed further in the text. 
Citation: Musubire AK, Boulware DR, Meya DB, Rhein J (2013) Diagnosis and Management of Cryptococcal Relapse. J AIDS Clinic Res S3: 003. doi:10.4172/2155-6113.S3-003

Page 3 of 8

\begin{tabular}{|l|l|}
\hline Paradoxical IRIS Criterion & Alternative diagnosis (if criterion not met) \\
\hline Prior diagnosis of cryptococcal infection & Possible Unmasking Cryptococcal Infection \\
\hline $\begin{array}{l}\text { Documented clinical improvement after initiation of appropriate antifungal } \\
\text { therapy and prior to suspected IRIS event }\end{array}$ & Primary failure of antifungal therapy \\
\hline $\begin{array}{l}\text { Receiving antiretroviral therapy (ART) } \\
\text { Virologic response with } \mathbf{> 1} \mathbf{~ l o g ~}_{10} \text { copies/mL decrease in HIV RNA (optional to } \\
\text { verify if compliant with ART) }\end{array}$ & $\begin{array}{l}\text { Progression of cryptococcosis due to ongoing immunosuppression, or } \\
\text { No suitable specimen available for exclusion of virologic failure }\end{array}$ \\
\hline $\begin{array}{l}\text { Clinical deterioration with an inflammatory condition } \\
\text { (e.g. meningitis, cryptococcomas, pneumonitis, lymphadenopathy, } \quad \text { or } \\
\text { cutaneous / soft tissue lesions) }\end{array}$ & Consider alternative pathogens and/or non-inflammatory pathology \\
\hline $\begin{array}{l}\text { Negative cryptococcal culture } \\
\text { Investigations negative for bacterial and mycobacterial infection }\end{array}$ & True culture-positive relapse of cryptococcosis during treatment \\
\hline Significant ART or antifungal non-adherence & Relapse of cryptococcosis due to non-adherence to therapy \\
\hline
\end{tabular}

Table 2:. Paradoxical IRIS Criteria [16].

\begin{tabular}{|l|l|l|l|l|}
\hline Reference & Country & Induction Regimen & Prevalence & Causes \\
\hline Jarvis [20] & Cape Town, South Africa & $\begin{array}{l}\text { Amphotericin } \\
\text { fluconazole }\end{array}$ & $+12.7 \%$ of 300 CM & Not taking fluconazole (79\%) \\
\hline Espie [41] & Cambodia & Amphotericin & $13.7 \%$ of $750 \mathrm{CM}$ & $14.1 \%$ overall not prescribed secondary prophylaxis \\
\hline Jongwutiwes [4] & Thailand & Amphotericin & $24.4 \%$ of 127 CM & No ART: 32\% relapsed. With ART: 13.5\% relapsed. \\
\hline Boulware [28] & Uganda & Amphotericin & $5 \%$ of 101 CM & $\begin{array}{l}\text { Resistance (40\%) } \\
\text { Unknown (60\%) }\end{array}$ \\
\hline Bicanic [14] & Cape Town, South Africa & Fluconazole 400mg & 21 episodes & $67 \%$ reduced fluconazole susceptibility \\
\hline Sachdeva [3] & India & Amphotericin & $10 \%$ of 91 CM & Not taking fluconazole (44\%), No ART (11\%), Unknown (44\%) \\
\hline Pitisuttithum [42] & Thailand & Amphotericin & $7.5 \%$ of 106 & N/A \\
\hline McCarthy [13] & Gauteng Prov., South Africa & Fluconazole primarily & $9.5 \%$ of 2753 & N/A \\
\hline
\end{tabular}

Table 3 : Prevalence of relapse.

infection. Relapse/treatment failure and CM-IRIS are not always mutually exclusive, just as drug resistant tuberculosis increases the risk of TB-IRIS [25].

Since there is no laboratory test available for detection of paradoxical CM-IRIS, IRIS remains primarily a clinical diagnosis of exclusion, with the principal consideration being exclusion of culture-positive relapse. A positive CSF culture for C. neoformans is diagnostic for microbiologic relapse or treatment failure, but positive CSF India ink by itself is not sufficient for determining relapse as the presence of lingering nonviable yeast in CSF is common. We have observed positive India ink stains in the setting of sterile CSF cultures up to a year after diagnosis. Both India ink and a qualitative cryptococcal antigen ( $\mathrm{CrAg}$ ) have zero diagnostic utility for distinguishing IRIS from relapse. Furthermore, CrAg titers are not precise indicators for early relapse or for making therapeutic decisions, though titers may be clinically helpful for determining late relapse [26,27]. CrAg titers generally decline gradually over months to years at variable rates. The $\mathrm{CrAg}$ change over time did not correlate with clinical outcome in the pre-ART era [27]. While not definitive, an unchanged or rising CrAg titer several months after initial CM diagnosis should raise one's suspicion for microbiologic relapse while one awaits the culture.

Another diagnostic clue can be the CSF WBC count. In a Kampala cohort, the median CSF WBC counts at time of CM-IRIS was higher (median 50, IQR: 5 to 117, max 212 cells/ $\mu \mathrm{L}$ ) than in CMrelapse (median 10, Range 5-30 cells $/ \mu \mathrm{L})(P=.044)$ [23]. As these CSF WBC overlap for IRIS and relapse, these CSF WBC counts are also not definitive. However, a low CSF WBC count should also raise one's suspicion of the possibility of relapse, whereas higher WBC counts are more apparent during IRIS events.

Other parameters and features are not overly helpful is distinguishing relapse from CM-IRIS. Shelburne et al. reported that persons with relapse returned later than patients with CM-IRIS (165 days vs. 59 days, $\mathrm{P}=0.029$ ) after $\mathrm{CM}$ diagnosis, were less likely to have initiated ART ( $33 \%$ vs. $100 \%, \mathrm{P}<0.001$ ), and less likely to have virological and immunological response to ART (median decrease in HIV-1 RNA level,-0.15 in relapse vs. $-2.27 \log _{10}$ copies $/ \mathrm{mL}$ in CMIRIS; P < 0.001; median increase in CD4+ cell count, 4 vs. 93 cells $/ \mu \mathrm{L}$; $\mathrm{P}=0.002$ ) [24]. This cohort included significant number of persons from the pre-ART era who may have had pre-existing virologic resistance. In a Ugandan 2006-2009 cohort previously ART-naive, virologic failure was also more common in relapse ( 3 of 5 relapses) than in IRIS (none); however, CD4 responses were similar. CD4 cells do not always increase with IRIS as $30 \%$ had $<25 \mathrm{CD} 4 \mathrm{cell} / \mu \mathrm{L}$ increase in their CD4 count at time of CM-IRIS event from their preART nadir $[23,28]$. In our experience, CD4 is not a useful diagnostic marker for either CM-IRIS or relapse [28].

The immune response differs at the time of an IRIS event compared to a culture-positive relapse. In the Ugandan prospective cohort study, the investigators measured the CSF cytokine profiles observed at time of a clinical relapse and compared the cytokine profile between IRIS events vs. culture-positive relapses. In a study with 85 subjects in which 33 (39\%) developed CM-related IRIS and 5 (6\%) developed culture-positive CM relapse, CSF parameters in persons with culture-positive CM relapse showed minimal signs of inflammation [23] compared with paradoxical CM-related IRIS, CSF from patients with CM culture-positive relapse had lower levels of the pro-inflammatory cytokines TNF- $\alpha(\mathrm{P}=0.022), \mathrm{IL}-17(\mathrm{P}=0.018)$, and Th1 cytokines of IFN- $\gamma(\mathrm{P}=0.004)$ and IL-12 $(\mathrm{P}=0.006)$, as well as the cytokines IL-9 $(\mathrm{P}<0.001)$ and Th2 cytokine IL-4 $(\mathrm{P}=0.005)$. Persons with relapse had markedly higher IL-13 levels ( $\mathrm{P}=0.04),[23]$, a Th2 cytokine, which is non-protective in cryptococcosis [29-31].

In order to obtain an accurate diagnosis, microbiology culture is the gold standard [5,32-35]. Imaging and fluconazole resistance 
testing in patients with recurrent meningitis may also be used in guiding treatment decisions [34,36-39]. Neither Crag titres nor CD4 counts are useful in differentiating between relapse and CM IRIS $[35,40]$.

\section{Prevalence}

The prevalence of relapse depended on the initial antifungal regimen, presence of ART, and/or adherence to secondary prophylaxis use (Table 3). In a study by Jarvis et al. [20] among 300 confirmed laboratory episodes of cryptococcal meningitis from South Africa, 69 were relapse episodes, accounting for $23 \%$ of all cases of cryptococcal meningitis. Approximately $43 \%$ (30) of relapse episodes were among patients not taking fluconazole secondary prophylaxis, $45 \%$ (31) were due to IRIS and $12 \%$ (8) were in patients taking fluconazole prior to ART initiation. These patients also received amphotericin $\mathrm{B}$ and fluconazole [20]. Espie et al. [41] looked at 750 patients from Cambodia who were in care after 3 months of CM diagnosis and of these $85.9 \%$ had received secondary prophylaxis and $13.7 \%$ relapsed at a median of 5.7 months (interquartile range 4.1-8.8). The initial antifungal regimen was amphotericin B followed by fluconazole consolidation and maintenance therapy. Boulware et al. [28] among 101 subjects with CM who started ART in Uganda, reported 5 (5\%) of patients developed culture-positive CM relapse [23,28]. Both studies used the same antifungal regimen. A prospective study involving Thai adults $(n=106)$ with AIDS-associated CM only had eight patients with relapse, of whom five died, two were lost to followup, and one survived for more than 1 year [42]. The median time to relapse was 21 days after last negative CSF culture. Later mortality in this study was associated with delayed CSF yeast clearance $(\mathrm{RR}=3.6$; $95 \% \mathrm{CI}, 1.9$ - 6.4) and relapse ( $\mathrm{RR}=3.9 ; 95 \% \mathrm{CI}, 1.4-10.8)$. A study in Gauteng Province, South Africa of 2753 incident cases of CM noted recurrences occurred in 263 (9.5\%) incident cases [13]. These data suggests that relapse rates can be anywhere between $6-23 \%$.

\section{Causes of Relapse}

There are multiple potential causes of relapse of cryptococcal infection including: the activity of the initial antifungal regimen, compliance with fluconazole secondary prophylaxis, and ART use. In cohorts using less active antifungal regimens, such as fluconazole, relapse occurred more frequently $[19,43]$ as well as frequently reduced fluconazole susceptibility. Bicanic et al. [14] have reported, two-thirds of persons with culture-positive relapse had fluconazole resistance in South Africa [14]. Poor adherence to fluconazole is another contributor to relapse $[20,44]$. This poor compliance may be related to health system issues including; failed prescriptions, dispensing, referral for or adherence to secondary fluconazole prophylaxis [20]. Relapses can also occur due to prolonged immune suppression in patients not taking ART $[4,20]$. The management of IRIS with corticosteroids may increase the risk of culture positive CM-relapse [34]. Drug interactions especially with rifampicin can potentially cause sub therapeutic levels of fluconazole that can theoretically cause relapse [14,45]. An Indian cohort, with 9 relapse cases among a total of 91 persons with CM, 8 of 9 patients relapsed $>6$ months after initiating ART and 5 patients had relapses beyond one year [3]. Among 5 patients, no reason for relapse could be identified [3]. Thus, the main causes of relapse are dependent on the initial induction therapy. For those receiving amphotericinbased regimens, which are microbiologically more active than azole regimens, [17] not receiving fluconazole secondary prophylaxis is a primary cause of relapse. In persons compliant with fluconazole or persons who received fluconazole induction therapy, fluconazole resistance must be suspected.

A lumbar puncture is performed in the clinic and the patient is hospitalized for further evaluation and management. His opening pressure is noted to be $>500 \mathrm{mmH}_{2} \mathrm{O}, \mathrm{CSF}$ WBC count is noted to be 255 cells $/ \mu \mathrm{L}$, and an India ink stain is positive. CSF is sent for quantitative cryptococcal cultures.

How does the above information change the differential diagnosis? How should this patient be managed?

\section{Management of Relapse}

Since differentiating relapse from CM-IRIS is dependent on CSF cultures, definitive diagnosis in suspected cases of relapse is typically delayed. For cases of suspected relapse, induction therapy should be reinstituted immediately, pending CSF culture. Re-induction therapy with high-dose amphotericin ( $1 \mathrm{mg} / \mathrm{kg} /$ day) for at least 7 days is often needed [46]. Fluconazole should also be increased back to induction doses (800-1200 mg/day). Higher doses of fluconazole are preferred in this situation as fluconazole resistance is a concern [14].

The patient is restarted on CM induction therapy with daily amphotericin and fluconazole is increased to $1200 \mathrm{mg} /$ daily. Serial lumbar punctures are initiated according to current guidelines. Four days after the initial lumbar puncture, cultures return positive for $C$. neoformans. Resistance testing is repeated and a minimum inhibitory concentration of $32 \mu \mathrm{g} / \mathrm{mL}$ is again measured for fluconazole. Opening pressure and quantitative CSF cultures for serial lumbar punctures from this hospitalization are summarized in Table 4.

The patient completes another 14-day course of amphotericin without complications. He is discharged on fluconazole $1200 \mathrm{mg} /$ day, and the patient is seen back in clinic 2 weeks later. CD4 count is 101 cells/ $\mu \mathrm{L}$ and an HIV viral load is undetectable. The CSF culture results from his day 14 culture are reviewed in clinic, and are noted to be positive for C. neoformans growth after 10 days of incubation, with a CSF quantitative colony count of $1.1 \times 10^{2} \mathrm{CFU} / \mathrm{mL}$ of CSF.

What are the possible causes of persistent infection in this case? Which diagnostic tests could help determine the cause? How should this be managed?

In cases of confirmed culture-positive CM relapse, induction therapy should be continued and isolates sent for fluconazole resistance testing. Antifungal susceptibility can be very useful at time of relapse to aid in decision making. Recurrences may be due to drug resistance of the original strain or an initial infection with a different strain or re infection with a new strain. In genotyping of serial $C$. neoformans isolates from 7 Cubans with recurrent cryptococcal meningitis, three patients had identical genotype isolates, and four had relapse isolates which were genotypically different from the initial infecting strain [47]. This has been demonstrated by other studies [48,49], including where new resistance has developed $[14,50,51]$. We have reported de-novo development of resistance with the same genotype of cryptococcal strain [34]. While interesting

\begin{tabular}{|c|c|c|}
\hline Day & Opening Pressure $\left(\mathrm{mm} \mathrm{H}_{2} \mathrm{O}\right)$ & Quantitative Colony Count $(\mathrm{CFU} / \mathrm{mL})$ \\
\hline 1 & $>500$ & $1.24 \times 10^{3}$ \\
\hline 3 & 420 & $2.9 \times 10^{2}$ \\
\hline 7 & 380 & $8 \times 10^{2}$ \\
\hline 14 & 180 & Pending \\
\hline
\end{tabular}

Table 4: CSF Parameters During Re-Hospitalization of Patient. 
Citation: Musubire AK, Boulware DR, Meya DB, Rhein J (2013) Diagnosis and Management of Cryptococcal Relapse. J AIDS Clinic Res S3: 003. doi:10.4172/2155-6113.S3-003

scientifically, the clinical concern is that relapse isolates may have decreased susceptibility to fluconazole.

For refractory disease, use of an alternative triazole such as voriconazole or weekly amphotericin should be considered, although comparative trials evaluating second-line therapy for CM relapse are limited. Voriconazole (300 $\mathrm{mg}$ twice daily) appeared to be as effective as flucytosine and high-dose fluconazole as adjunctive therapy with amphotericin in a randomized trial in South Africa [52]. Voriconazole has been successfully used in the treatment of refractory disease, particularly in the context of secondary fluconazole resistance [5357]. In addition to the high cost and limited availability, interactions between voriconazole and rifamycin [58] or ART [59], thereby limit voriconazole's use in resource-limited settings.

Other adjunctive antifungal therapies that have been used in the primary treatment of CM could also show promise for relapse disease, though clinical data for this purpose is currently lacking [60]. Adjunctive recombinant interferon $-\gamma\left(100 \mu \mathrm{g} / \mathrm{m}^{2} \times 2\right.$ doses $)$, for instance, was well tolerated and significantly increased the rate of cryptococcal clearance from CSF in a randomized trial from South Africa [61]. More recently, the selective serotonin receptor inhibitor (SSRI) sertraline was shown to provide potent in vitro fungicidal activity against $C$. neoformans [62]. In addition to its antifungal properties, sertraline holds promise in the treatment of CM since it is well tolerated with few drug-drug interactions, appears to have a novel mechanism of action, readily crosses the blood-brain barrier, has an excellent safety profile, and is widely available in an inexpensive, generic formulation. A randomized trial is set to commence in Uganda to evaluate the adjunctive use of sertraline in human cryptococcal meningitis.

Until new antifungals are developed, further evaluated, and become more widely accessible, however, clinicians must continue to rely on currently available induction therapy for treatment of relapse disease. The ability of amphotericin to rapidly and consistently sterilize the CSF of patients with CM suggests that it should be central to any re-induction strategy [19]. Most guidelines suggest that longer courses (4-10 weeks) of induction therapy should be reinstituted for relapse disease, [18] though the duration and timing of amphotericin must often be individualized according to the health of the patient, the development of amphotericin toxicity, patient proximity to hospital or clinic, and individual preference. The underlining goal, however, should always remain achievement of CSF sterility. Follow-up LPs to evaluate for fungal clearance are a necessity, but the frequency must be thoughtfully negotiated with the patient in advance in order to minimize the risk of the patient being lost to follow-up.

After refusing further hospitalization, the patient agrees to outpatient amphotericin infusions three times a week. 2 weeks later, the patient is sent for head computed tomography (CT) for further evaluation of persistent central nervous system (CNS) infection. Unfortunately he experiences a witnessed seizure shortly after receiving a CT (Figure 2), and he is hospitalized for further evaluation.

His seizures are controlled with anti-epileptics while hospitalized. Daily amphotericin is continued along with high dose fluconazole at $1200 \mathrm{mg} /$ day. Serology for toxoplasmosis is negative. A repeat CT suggests persistent lesions with worsening edema and possible masseffect and corticosteroids are initiated.

What are the possible causes of the patient's seizures? How should this be managed?

The most important late complications to consider in refractory cryptococcal disease include ongoing IRIS and cerebral cryptococcoma. In the context of ART-driven immune reconstitution, these conditions may exist concurrently. In this case, the presence of ongoing, low-grade treatment-refractory infection in the form of cerebral cryptococcoma is likely to have provided a source for an ongoing antigenic stimulation with a (dysregulated) immune response. The management of these late complications can be complex, especially when they occur together [34].

No detailed guidelines currently exist regarding the optimal medical management of cryptococcal IRIS. Control of intracranial pressure should be stressed, though in the context of known CNS focal lesions, care must be taken to rule out mass-effect prior to lumbar puncture to avoid the risk of herniation. Specific recommendations regarding the medical management of IRIS are lacking and decisions must be individualized according to the clinical presentation. Discontinuation of antiretroviral therapy is rarely necessary $[28,63]$. Corticosteroids, are at times utilized in severe cases of paradoxical cryptococcal IRIS with reported anecdotal benefit, [64,65] although steroids alone in the setting of persistent infection/relapse with fluconazole-resistance Cryptococcus can cause harm [34]. In our case, the decision to initiate steroids was based on the combination of suspected ongoing IRIS and CNS mass-effect with surrounding edema. In the setting of relapse, initiating steroids while giving amphotericin, pending a CSF culture, is not known to cause harm [14].
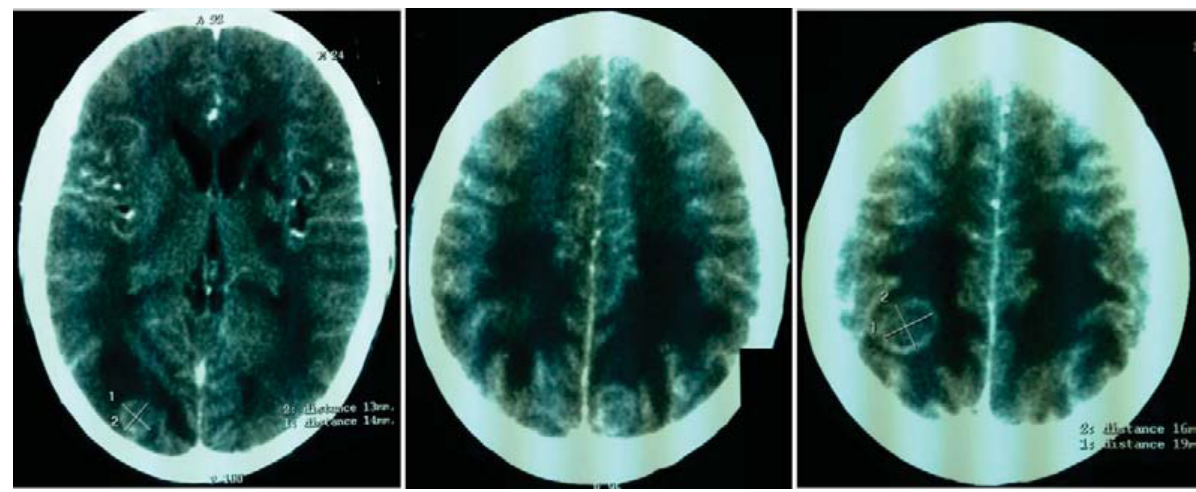

Figure 2: Head CT of patient with CM relapse.

Contrast-enhanced head CT of patient following his second hospitalization. Multiple round, sharply marginated, rim-enhancing lesions are apparent 
Citation: Musubire AK, Boulware DR, Meya DB, Rhein J (2013) Diagnosis and Management of Cryptococcal Relapse. J AIDS Clinic Res S3: 003. doi:10.4172/2155-6113.S3-003

Cerebral cryptococcomas be difficult to treat, generally requiring prolonged antifungal therapy. As with other complications related to $\mathrm{CM}$, optimization of antifungal drugs and the relief of increased intracranial pressure are central to management. As cryptococcomas are more common in immune competent individuals, [66] it follows that clinically relevant lesions are more likely to present as a late complication of ART-mediated immune reconstitution in persons living with AIDS. As with CM relapse without the development of cryptococcomas, extended periods of induction therapy are recommended, and the duration of treatment should be directed by mycological response rather than repeat imaging alone, which can be misleading [67]. In this patient with mass-effect and significant surrounding edema, a short trial of corticosteroids may be considered, though this is based on expert opinion rather than specific data. Surgery may be needed in life-threatening cases of mass-effect or to achieve cure in extremely prolonged cases.

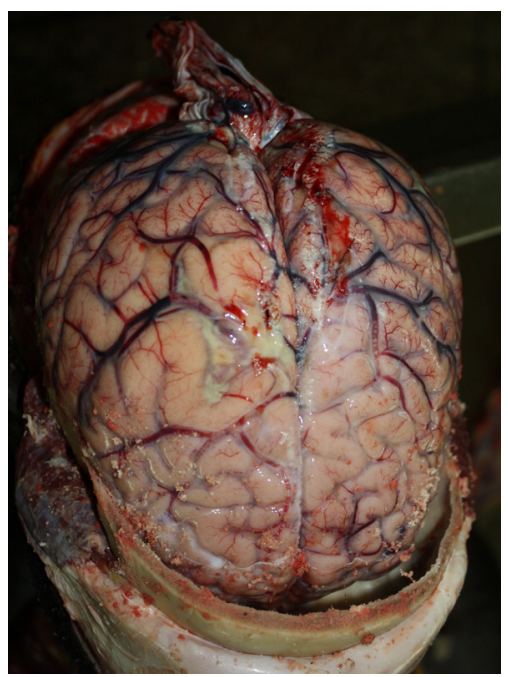

Figure 3a. The photograph shows the gross appearance of the brain within the cranial cavity following sawing of the skull cap. There is a scanty purulent exudate seen in the subarachnoid space (black arrow). The surface vessels are congested and the brain is visibly swollen showing signs of increased intracranial pressure.

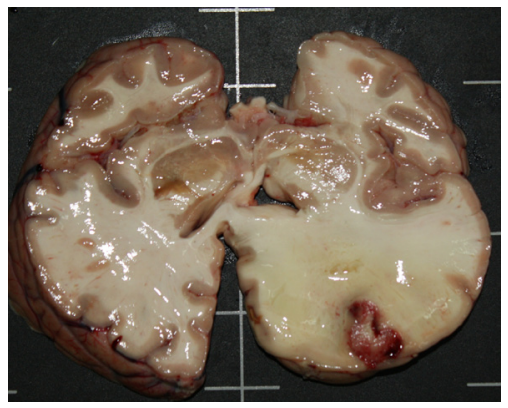

Figure 3b: The photograph shows coronal sections of the cerebral hemispheres. Note the focus of necrosis (purple arrow) with surrounding hyperemia and oedema (blue outline).

The histopathological examination of the brain showed mixed aetiology meningoencephalitis (acute bacterial infection and Cryptococcus yeast forms). A focal area in the parenchymal corresponding with a cryptococcoma showed surrounding gliosis and hemorrhage. The yeast forms stained positive with PAS stain. The ZN stain was negative.
Repeat CT imaging 7 days into the patient's hospitalization suggests improvement in mass-effect with decreased edema, and an LP is repeated with an opening pressure of $270 \mathrm{~mm} \mathrm{H}_{2} \mathrm{O}$. Positive growth of $C$. neoformans is noted 4 days later, with a quantitative colony count of $120 \mathrm{CFU} / \mathrm{mL}$ of CSF despite ongoing antifungal therapy. Note that the patient had received roughly 50 doses of amphotericin by this point ( $2.5 \mathrm{~g}$ cumulative dose). Corticosteroids begin to be tapered off.

After seizure-free ongoing improvement for a period of two weeks, the patient suddenly develops status epilepticus with rapid deterioration. He is transferred to intensive care but regrettably dies the next day. His family agrees to an autopsy.

Gross pathology reveals leptomeninges clouded by scanty purulent exudates over the surface of both hemispheres, features of elevated intracranial pressure, and a $4 \mathrm{~cm}$ necrotic lesion with surrounding edema in the parietal region of the left cerebral hemisphere (Figure 3 courtesy of Dr. Robert Lukande). Histopathology suggests meningitis with inflammatory infiltrate, predominantly polymorphs and cryptococcal yeast also present, in addition to a large abscess surrounded by yeast. Brain specimens grow Cryptococcus neoformans.

Final Diagnosis: Culture-positive Cryptococcoma complicated by immune reconstitution inflammatory syndrome

\section{Conclusion}

We have discussed a patient who presented with cryptococcal relapse, subsequently complicated by a cryptococcoma with viable organisms and IRIS. This review highlighted the clinical complexities in the diagnosis and management of Cryptococcal relapse. To minimize the probability of relapse, the key cornerstones of care are: improved antifungal induction with the amphotericin, achieving CSF sterility, secondary fluconazole prophylaxis, and ART initiation. . Despite these successes, culture-positive relapse should still be suspected in patients that present with supporting clinical presentation, particularly in those who have received fluconazole monotherapy for induction and have a paucity of CSF inflammation. The gold standard for diagnosis of relapse is culture positive CSF after a documented sterile CSF. CSF culture is essential while other testing such as CSF India ink, qualitative $\mathrm{CrAg}$, or $\mathrm{CD} 4$ count change are absolutely not diagnostically helpful for differentiating relapse from IRIS. Thus, although not recommended (as yet) by any guideline, we recommend continuing fluconazole at 800 $\mathrm{mg} /$ day through the first two weeks of consolidation therapy until the CSF is known to be sterile.

There is however areas in relapse that need further research. There are a few studies that have reflected the role of resistance testing and its implementation. The role of primary and secondary prophylaxis in development of future resistance has not been adequately studied. There are also issues with clinical management of refractory cryptococcal disease when limited options are available. There is also a challenge of concomitant presentation of culture-positive relapse and paradoxical CM-IRIS

\section{Acknowledgements}

We thank Dr. Robert Lukande for excellent pathology support which has assisted to improve patient care. No specific support funded this work; however authors are supported via U.S. National Institutes of Health U01AI089244, R21NS065713, T32AI055433, and K23AI073192.

\section{References}

1. Lee SJ, Choi HK, Son J, Kim KH, Lee SH (2011) Cryptococcal meningitis in patients with or without human immunodeficiency virus: experience in a tertiary hospital. Yonsei Med J 52: 482-487. 
Citation: Musubire AK, Boulware DR, Meya DB, Rhein J (2013) Diagnosis and Management of Cryptococcal Relapse. J AIDS Clinic Res S3: 003. doi:10.4172/2155-6113.S3-003

2. Laman M, Hwaihwanje I, Davis TM, Manning L (2010) Cryptococcal meningitis in immunocompetent Papua New Guinean children. Trop Doct 40: 61-63.

3. Sachdeva RK, Randev S, Sharma A, Wanchu A, Chakrabarti A, et al. (2012) A retrospective study of AIDS-associated cryptomeningitis. AIDS Res Hum Retroviruses 28: 1220-1226.

4. Jongwutiwes U, Kiertiburanakul S, Sungkanuparph S (2007) Impact of antiretroviral therapy on the relapse of cryptococcosis and survival of HIVinfected patients with cryptococcal infection. Curr HIV Res 5: 355-360.

5. Perfect JR, Dismukes WE, Dromer F, Goldman DL, Graybill JR, et al. (2010) Clinical practice guidelines for the management of cryptococcal disease: 2010 update by the infectious diseases society of america. Clin Infect Dis 50: 291322.

6. Becquet R, Ekouevi DK, Arrive E, Stringer JS, Meda N, et al. (2009) Universal antiretroviral therapy for pregnant and breast-feeding HIV-1-infected women: towards the elimination of mother-to-child transmission of HIV-1 in resourcelimited settings. Clin Infect Dis 49: 1936-1945.

7. Uebel KE, Timmerman V, Ingle SM, van Rensburg DH, Mollentze WF (2010) Towards universal ARV access: achievements and challenges in Free State Province, South Africa. S Afr Med J 100: 589-593.

8. Campbell C, Scott K, Madenhire C, Nyamukapa C, Gregson S (2011) Sources of motivation and frustration among healthcare workers administering antiretroviral treatment for HIV in rural Zimbabwe. AIDS Care 23: 797-802.

9. Berhe T, Melkamu Y, Amare A (2012) The pattern and predictors of mortality of HIVIAIDS patients with neurologic manifestation in Ethiopia: a retrospective study. AIDS Res Ther 9: 11

10. Amuron B, Levin J, Birunghi J, Namara G, Coutinho A, et al. (2011) Mortality in an antiretroviral therapy programme in Jinja, south-east Uganda: a prospective cohort study. AIDS Res Ther 8: 39.

11. Lee YC, Wang JT, Sun HY, Chen YC (2011) Comparisons of clinical features and mortality of cryptococcal meningitis between patients with and without human immunodeficiency virus infection. J Microbiol Immunol Infect 44: 338345

12. Zhu LP, Wu JQ, Xu B, Ou XT, Zhang QQ, et al. (2010) Cryptococcal meningitis in non-HIV-infected patients in a Chinese tertiary care hospital, 1997-2007. Med Mycol 48: 570-579.

13. McCarthy KM, Morgan J, Wannemuehler KA, Mirza SA, Gould SM, et al. (2006) Population-based surveillance for cryptococcosis in an antiretroviral-naive South African province with a high HIV seroprevalence. AIDS 20: 2199-2206.

14. Bicanic T, Harrison T, Niepieklo A, Dyakopu N, Meintjes G (2006) Symptomatic relapse of HIV-associated cryptococcal meningitis after initial fluconazole monotherapy: the role of fluconazole resistance and immune reconstitution. Clin Infect Dis 43: 1069-1073.

15. Mónaco LS, Tamayo Antabak N (2008) [Cryptococcosis in AIDS patients: case study from 1996 to 2006 in Paroissien Hospital]. Rev Argent Microbiol 40: 218221

16. Haddow LJ, Colebunders R, Meintjes G, Lawn SD, Elliott JH, et al. (2010) Cryptococcal immune reconstitution inflammatory syndrome in HIV-1-infected individuals: proposed clinical case definitions. Lancet Infect Dis 10: 791-802.

17. Rhein J, Boulware DR (2012) Prognosis and management of cryptococcal meningitis in patients with human immunodeficiency virus infection. Neurobehav HIV Med 4: 45-61.

18. Perfect JR, Dismukes WE, Dromer F, Goldman DL, Graybill JR, et al. (2010) Clinical practice guidelines for the management of cryptococcal disease: 2010 update by the infectious diseases society of america. Clin Infect Dis 50: 291 322

19. Bicanic T, Meintjes G, Wood R, Hayes M, Rebe K, et al. (2007) Fungal burden, early fungicidal activity, and outcome in cryptococcal meningitis in antiretroviralnaive or antiretroviral-experienced patients treated with amphotericin B or fluconazole. Clin Infect Dis 45: 76-80.

20. Jarvis JN, Meintjes G, Williams Z, Rebe K, Harrison TS (2010) Symptomatic relapse of HIV-associated cryptococcal meningitis in South Africa: the role of inadequate secondary prophylaxis. S Afr Med J 100: 378-382.

21. Aller Al, Martin-Mazuelos E, Lozano F, Gomez-Mateos J, Steele-Moore L, et al. (2000) Correlation of fluconazole MICs with clinical outcome in cryptococcal infection. Antimicrob Agents Chemother 44: 1544-1548.
22. Brion LP, Uko SE, Goldman DL (2007) Risk of resistance associated with fluconazole prophylaxis: systematic review. J Infect 54: 521-529.

23. Boulware DR, Bonham SC, Meya DB, Wiesner DL, Park GS, et al. (2010) Paucity of initial cerebrospinal fluid inflammation in cryptococcal meningitis is associated with subsequent immune reconstitution inflammatory syndrome. $J$ Infect Dis 202: 962-970.

24. Shelburne SA 3rd, Darcourt J, White AC Jr, Greenberg SB, Hamill RJ, et al. (2005) The role of immune reconstitution inflammatory syndrome in AIDS-related Cryptococcus neoformans disease in the era of highly active antiretroviral therapy. Clin Infect Dis 40: 1049-1052.

25. Meintjes G, Rangaka MX, Maartens G, Rebe K, Morroni C, et al. (2009) Novel relationship between tuberculosis immune reconstitution inflammatory syndrome and antitubercular drug resistance. Clin Infect Dis 48: 667-676.

26. Powderly WG, Cloud GA, Dismukes WE, Saag MS (1994) Measurement of cryptococcal antigen in serum and cerebrospinal fluid: value in the management of AIDS-associated cryptococcal meningitis. Clin Infect Dis 18: 789-792.

27. Aberg JA, Watson J, Segal M, Chang LW (2000) Clinical utility of monitoring serum cryptococcal antigen (sCRAG) titers in patients with AIDS-related cryptococcal disease. HIV Clin Trials 1: 1-6.

28. Boulware DR, Meya DB, Bergemann TL, Wiesner DL, Rhein J, et al. (2010) Clinical features and serum biomarkers in HIV immune reconstitution inflammatory syndrome after cryptococcal meningitis: a prospective cohor study. PLoS Med 7: e1000384.

29. Muller U, Stenzel W, Kohler G, Werner C, Polte T, et al. (2007) IL-13 induces disease-promoting Type 2 cytokines, alternatively activated macrophages and allergic inflammation during pulmonary infection of mice with Cryptococcus neoformans. J Immunol 179: 5367-5377.

30. Stenzel W, Müller U, Köhler G, Heppner FL, Blessing M, et al. (2009) IL-4/ IL-13-dependent alternative activation of macrophages but not microglial cells is associated with uncontrolled cerebral cryptococcosis. Am J Pathol 174: 486496.

31. Voelz K, Lammas DA, May RC (2009) Cytokine signaling regulates the outcome of intracellular macrophage parasitism by Cryptococcus neoformans. Infect Immun 77: 3450-3457.

32. Seilmaier M, Hecht A, Guggemos W, Rüdisser K (2009) [Cryptococcal meningoencephalitis related to HIV infection with resistance to fluconazole, relapse, and IRIS]. Med Klin (Munich) 104: 58-62.

33. Schulze K, Schmiedel S, van Lunzen J (2012) [Immune reconstitution inflammatory syndrome in cryptococcal meningitis: a rare phenomenon?] Dtsch Med Wochenschr 137: 834-837.

34. Musubire AK, Meya BD, Mayanja-Kizza H, Lukande $\mathrm{R}$, Wiesner LD, et al. (2012) Challenges in diagnosis and management of Cryptococcal immune reconstitution inflammatory syndrome (IRIS) in resource limited settings. Afr Health Sci 12: 226-230.

35. Cinti SK, Armstrong WS, Kauffman CA (2001) Case report. Recurrence of increased intracranial pressure with antiretroviral therapy in an AIDS patient with cryptococcal meningitis. Mycoses 44: 497-501.

36. Legris T, Massad M, Purgus R, Vacher-Coponat $H$, Ranque $S$, et al. (2011) Immune reconstitution inflammatory syndrome mimicking relapsing cryptococcal meningitis in a renal transplant recipient. Transpl Infect Dis 13: 303-308.

37. da Cunha Colombo ER, Mora DJ, Silva-Vergara ML (2011) Immune reconstitution inflammatory syndrome (IRIS) associated with Cryptococcus neoformans infection in AIDS patients. Mycoses 54: e178-182.

38. York J, Bodi I, Reeves I, Riordan-Eva P, Easterbrook PJ (2005) Raised intracranial pressure complicating cryptococcal meningitis: immune reconstitution inflammatory syndrome or recurrent cryptococcal disease? J Infect 51: 165-171

39. Boelaert JR, Goddeeris KH, Vanopdenbosch LJ, Casselman JW (2004) Relapsing meningitis caused by persistent cryptococcal antigens and immune reconstitution after the initiation of highly active antiretroviral therapy. AIDS 18 : 1223-1224.

40. Seddon J, Mangeya N, Miller RF, Corbett EL, Ferrand RA (2009) Recurrence of cryptococcal meningitis in HIV-infected patients following immune reconstitution. Int J STD AIDS 20: 274-275. 
Citation: Musubire AK, Boulware DR, Meya DB, Rhein J (2013) Diagnosis and Management of Cryptococcal Relapse. J AIDS Clinic Res S3: 003. doi:10.4172/2155-6113.S3-003

41. Espié E, Pinoges L, Balkan S, Chanchhaya N, Molfino L, et al. (2010) Cryptococcal meningitis in HIV-infected patients: a longitudinal study in Cambodia. Trop Med Int Health 15: 1375-1381.

42. Pitisuttithum P, Tansuphasawadikul S, Simpson AJ, Howe PA, White NJ (2001) A prospective study of AIDS-associated cryptococcal meningitis in Thailand treated with high-dose amphotericin B. J Infect 43: 226-233.

43. Mussini C, Pezzotti P, Miró JM, Martinez E, de Quiros JC, et al. (2004) Discontinuation of maintenance therapy for cryptococcal meningitis in patients with AIDS treated with highly active antiretroviral therapy: an international observational study. Clin Infect Dis 38: 565-571.

44. Kumar S, Wanchu A, Chakrabarti A, Sharma A, Bambery P, et al. (2008) Cryptococcal meningitis in HIV infected: experience from a North Indian tertiary center. Neurol India 56: 444-449.

45. Panomvana Na Ayudhya D, Thanompuangseree N, Tansuphaswadikul S (2004) Effect of rifampicin on the pharmacokinetics of fluconazole in patients with AIDS. Clin Pharmacokinet 43: 725-732.

46. Bicanic T, Wood R, Meintjes G, Rebe K, Brouwer A, et al. (2008) High-dose amphotericin $\mathrm{B}$ with flucytosine for the treatment of cryptococcal meningitis in HIV-infected patients: a randomized trial. Clin Infect Dis 47: 123-130.

47. Illnait-Zaragozi MT, Martinez-Machin GF, Fernandez-Andreu CM, Hagen F, Boekhout T, et al. (2010) Microsatellite typing and susceptibilities of seria Cryptococcus neoformans isolates from Cuban patients with recurrent cryptococcal meningitis. BMC Infect Dis 10: 289.

48. Mora DJ, Pedrosa AL, Rodrigues V, Leite Maffei CM, Trilles L, et al. (2010) Genotype and mating type distribution within clinical Cryptococcus neoformans and Cryptococcus gattii isolates from patients with cryptococcal meningitis in Uberaba, Minas Gerais, Brazil. Med Mycol 48: 561-569.

49. Yuanjie Z, Julin G, Fubing C, Jianghan C (2008) Recurrent pulmonary cryptococcosis in a patient with idiopathic CD4 lymphocytopenia. Med Mycol 46: 729-734.

50. Assing K, Birgens H, Arendrup M (2003) Cryptococcus neoformans var neoformans resistant to fluconazole in an HIV-negative patient with chronic lymphocytic leukemia. Clin Microbiol Infect 9: 441-444.

51. Blasi E, Brozzetti A, Francisci D, Neglia R, Cardinali G, et al. (2001) Evidence of microevolution in a clinical case of recurrent Cryptococcus neoformans meningoencephalitis. Eur J Clin Microbiol Infect Dis 20: 535-543.

52. Loyse A, Wilson D, Meintjes G, Jarvis JN, Bicanic T, et al. (2012) Comparison of the early fungicidal activity of high-dose fluconazole, voriconazole, and flucytosine as second-line drugs given in combination with amphotericin B for the treatment of HIV-associated cryptococcal meningitis. Clin Infect Dis 54: 121-128.

53. Sabbatani S, Manfredi R, Pavoni M, Consales A, Chiodo F (2004) Voriconazole proves effective in long-term treatment of a cerebral cryptococcoma in a chronic nephropathic HIV-negative patient, after fluconazole failure. Mycopathologia 158: $165-171$.
54. Chang HH, Lee NY, Ko WC, Lee HC, Yang YH, et al. (2010) Voriconazole inhibition of tacrolimus metabolism in a kidney transplant recipient with fluconazole-resistant cryptococcal meningitis. Int J Infect Dis 14: e348-350.

55. Perfect JR, Marr KA, Walsh TJ, Greenberg RN, DuPont B, et al. (2003) Voriconazole treatment for less-common, emerging, or refractory fungal infections. Clin Infect Dis 36: 1122-1131.

56. Nierenberg NE, Thompson GR, Lewis JS, Hogan BK, Patterson TF (2010) Voriconazole use and pharmacokinetics in combination with interferon-gamma for refractory cryptococcal meningitis in a patient receiving low-dose ritonavir. Med Mycol 48: 532-536.

57. Carbonara S, Regazzi M, Ciracì E, Villani P, Stano F, et al. (2009) Long-term efficacy and safety of TDM-assisted combination of voriconazole plus efavirenz in an AIDS patient with cryptococcosis and liver cirrhosis. Ann Pharmacother 43: $978-984$.

58. Brüggemann RJ, Alffenaar JW, Blijlevens NM, Billaud EM, Kosterink JG, et al (2009) Clinical relevance of the pharmacokinetic interactions of azole antifungal drugs with other coadministered agents. Clin Infect Dis 48: 1441-1458.

59. Yakiwchuk EM, Foisy MM, Hughes CA (2008) Complexity of interactions between voriconazole and antiretroviral agents. Ann Pharmacother 42: 698-703.

60. Pappas PG, Bustamante B, Ticona E, Hamill RJ, Johnson PC, et al. (2004) Recombinant interferon- gamma $1 \mathrm{~b}$ as adjunctive therapy for AIDS-related acute cryptococcal meningitis. J Infect Dis 189: 2185-2191.

61. Jarvis JN, Meintjes G, Rebe K, Williams GN, Bicanic T, et al. (2012) Adjunctive interferon- $\hat{I}^{3}$ immunotherapy for the treatment of HIV-associated cryptococcal meningitis: a randomized controlled trial. AIDS 26: 1105-1113.

62. Zhai B, Wu C, Wang L, Sachs MS, Lin X (2012) The antidepressant sertraline provides a promising therapeutic option for neurotropic cryptococcal infections. Antimicrob Agents Chemother 56: 3758-3766.

63. Bicanic T, Muzoora C, Brouwer AE, Meintjes G, Longley N, et al. (2009) Independent association between rate of clearance of infection and clinical outcome of HIV-associated cryptococcal meningitis: analysis of a combined cohort of 262 patients. Clin Infect Dis 49: 702-709.

64. Lortholary O, Fontanet A, Mémain N, Martin A, Sitbon K, et al. (2005) Incidence and risk factors of immune reconstitution inflammatory syndrome complicating HIV-associated cryptococcosis in France. AIDS 19: 1043-1049.

65. Huits RM, Bremmer R, Enting RH, Sprenger HG, van Assen S (2007) Return of meningeal symptoms in a patient treated for cryptococcal meningitis. J Neurol 254: $1443-1444$

66. Chen S, Sorrell T, Nimmo G, Speed B, Currie B, et al. (2000) Epidemiology and host- and variety-dependent characteristics of infection due to Cryptococcus neoformans in Australia and New Zealand. Australasian Cryptococcal Study Group. Clin Infect Dis 31: 499-508.

67. Hospenthal DR, Bennett JE (2000) Persistence of cryptococcomas on neuroimaging. Clin Infect Dis 31: 1303-1306. 